\title{
A SiGe HEMT Mixer IC with Low Conversion Loss
}

\author{
I. Kallfass, F. Gruson, P. Abele, K. Michelakis*, T. Hackbarth**, K.-H. Hieber**, J. Müller**, \\ H. Schumacher
}

\author{
Department of Electron Devices and Circuits, University of Ulm \\ Albert-Einstein-Allee 45, 89081 Ulm, Germany \\ ikall@ebs.e-technik.uni-ulm.de \\ * Department of Electrical and Electronic Engineering, Imperial College London \\ London SW7 2AZ, UK \\ ** DaimlerChrysler Research Centre, Wilhelm-Runge-Straße 11, 89013 Ulm, Germany
}

\begin{abstract}
The authors present the first SiGe HEMT mixer integrated circuit. The active mixer stage, operating up to $10 \mathrm{GHz} \mathrm{RF}$, has been designed and realized using a $0.1 \mu \mathrm{m}$ gate length transistor technology. The design is based on a new large-signal simulation model developed for the SiGe HEMT. Good agreement between simulation and measurement is reached. The mixer exhibits $4.0 \mathrm{~dB}$ and $4.7 \mathrm{~dB}$ conversion loss when down-converting $3.0 \mathrm{GHz}$ and 6.0GHz signals, respectively, to an intermediate frequency of $500 \mathrm{MHz}$ using high-side injection of $5 \mathrm{dBm}$ local oscillator power. Conversion loss is less than $8 \mathrm{~dB}$ for RF frequencies up to $10 \mathrm{GHz}$ with a mixer linearity of $\mathbf{- 8 . 8 \mathrm { dBm }}$ input related $1 \mathrm{~dB}$ compression point.
\end{abstract}

\section{INTRODUCTION}

The High Electron Mobility Transistor (HEMT) concept today offers the highest transistor cut-off frequencies combined with power capability and low noise performance. These devices are well established on GaAs and InP substrates but their relatively high production cost makes them less attractive for many consumer applications. Realizing HEMT structures on Si substrates combines HEMT performance with low cost and the possibility of integration with other Si-based technologies. Such devices use the high mobility of electrons in a two-dimensional electron gas (2DEG) in a strained $\mathrm{Si}$ layer. Successful realization has been demonstrated with respectable device performance [1][4]. A transimpedance amplifier IC using the SiGe HEMT has been reported in [5]. However, its design was not based on simulation due to the lack of models. As a consequence, the next step towards realization of integrated circuit prototypes in SiGe HEMT technology was the development of a simulation model. An empirical large-signal model has been demonstrated in [6]. Based on these prerequisites, the first SiGe HEMT mixer IC has been designed, processed and characterized. The design of an active mixer is, among other applications, well suited for demonstrating the technology's capabilities. It makes use of the transistor's high frequency- as well as low noise performance. The high linearity inherent to HEMT technology will be advantageous for good mixer linearity. Also, gain requirements are less stringent in the design of mixers.

The contribution presents simulation and measurement results of an active mixer cell designed with $0.1 \mu \mathrm{m}$ gate length transistors. After a brief review of device features and the development of a large-signal simulation model for the SiGe HEMT, the mixer circuit will be discussed in detail.

\section{SIGE HEMT TECHNOLOGY}

In the SiGe HEMT, a 2DEG forms in a strained Si layer sandwiched between two n-doped SiGe supply layers [7]. In order to form relaxed SiGe layers, a graded buffer is grown on the Si wafer. The gate is formed by a Schottkytype contact. It has a length of $100 \mathrm{~nm}$ and is centred between the source and drain implant areas which have a spacing of $1 \mu \mathrm{m}$. The device exhibits $\mathrm{n}$-channel depletion mode behaviour with a threshold voltage of typically $\mathrm{V}_{\text {th }}=-0.7 \mathrm{~V}$. Although devices with much higher $\mathrm{f}_{\mathrm{T}} / \mathrm{f}_{\max }$ have been demonstrated [2], the transistors used for model extraction show a maximum $\mathrm{f}_{\mathrm{T}}$ of $50 \mathrm{GHz}$ and $\mathrm{f}_{\max }$ of $80 \mathrm{GHz}$ (defined via Mason's unilateral gain). Maximum transconductance is $240 \mathrm{mS} / \mathrm{mm}$.

\section{LARGE-SIGNAL MODEL}

The design of mixer circuits requires a large-signal simulation model, which accurately describes the nonlinearities in the devices responsible for the generation of the frequency translating effect. The model of the SiGe HEMT is based on a typical FET equivalent circuit and uses empirical equations to describe the nonlinear elements.

The IV model employs the COBRA equation [8] developed at the National University of Ireland, Dublin, to describe the static drain current source $I_{D S}$ (Figure 1). Other non-linear model elements are Schottky-gate 
diodes, gate-source- and gate-drain capacitance as well as an $I_{d s}$ current correction source accounting for lowfrequency dispersion effects.

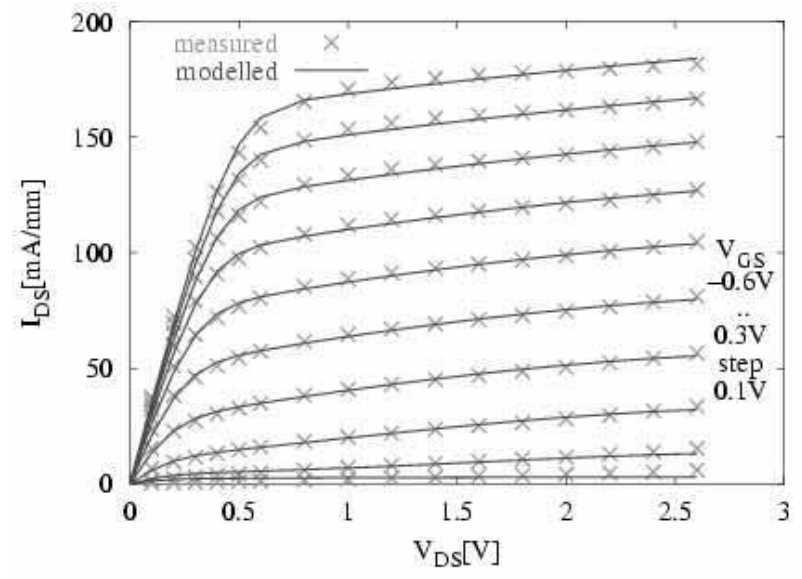

Figure 1: DC-IV model verification of a $0.1 \mu \mathrm{m}$ gate length, $2 \times 50 \mu \mathrm{m}$ width SiGe HEMT transistor.

The dynamic device model is based on analytical extraction of the small-signal model parameters at bias points over the whole IV plane [9] [10]. Figure 2 shows the hereby obtained voltage dependence of $\mathrm{C}_{\mathrm{gs}}$ and $\mathrm{C}_{\mathrm{gd}}$. Empirical expressions are employed to model the nonlinear gate capacitances.
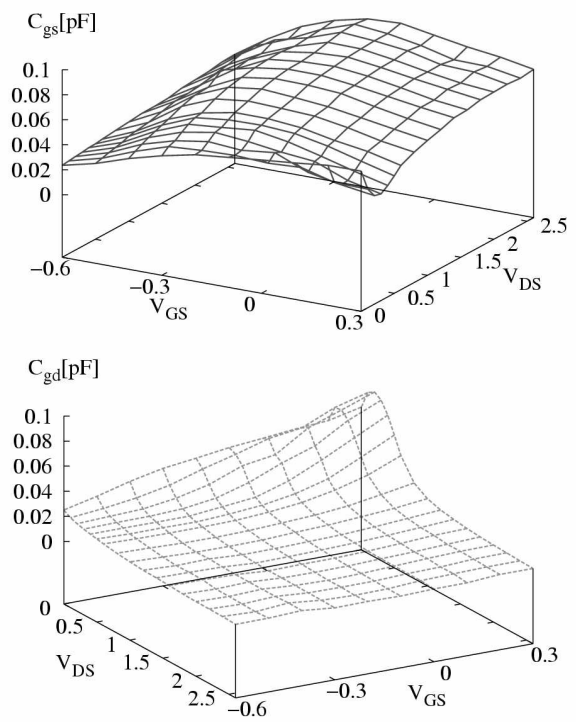

Figure 2 : Gate-Source (a) and Gate-Drain (b) capacitance.

Each grid intersection represents an extraction point

LF dispersion effects play an important role in these devices, introducing frequency dependence to trans- and output conductance, and need to be included in the model in order to predict high-frequency behaviour. The dynamic large-signal $\mathrm{I}_{\mathrm{ds}}$ characteristic is obtained through simultaneous optimisation of the device's trans- and output conductance extracted at high frequencies.
The model is implemented in the ADS simulation environment and can be verified against S-parameter measurements (Figure 3). It is suitable for all types of simulations, ranging from small-signal to full largesignal analyses in the time- and frequency domain.

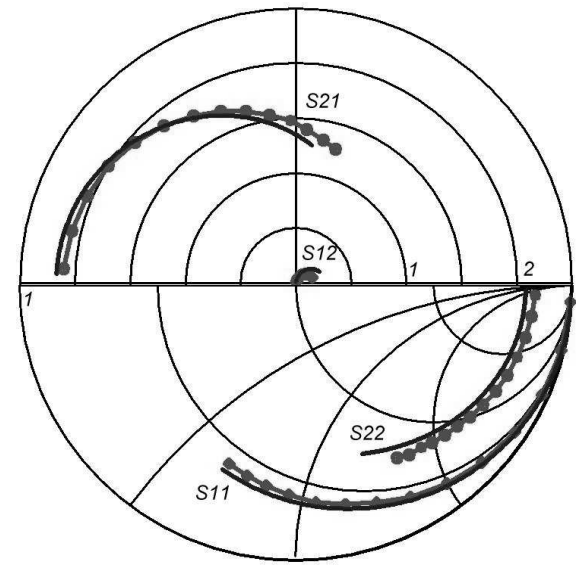

Figure 3 : Comparison of measured and modelled $S$ parameters up to $40 \mathrm{GHz}$ for a typical bias point in saturation. Dotted lines are measured values.The simulation employs the complete large-signal FET model.

\section{MIXER CIRCUIT}

The presented mixer circuit uses a single-balanced Gilbert cell topology, with the differential LO signal being applied to the upper transistors of a differential stage (Figure 4), which act as current switches. The lower transistor acts as a common-source amplifier to the RF signal.

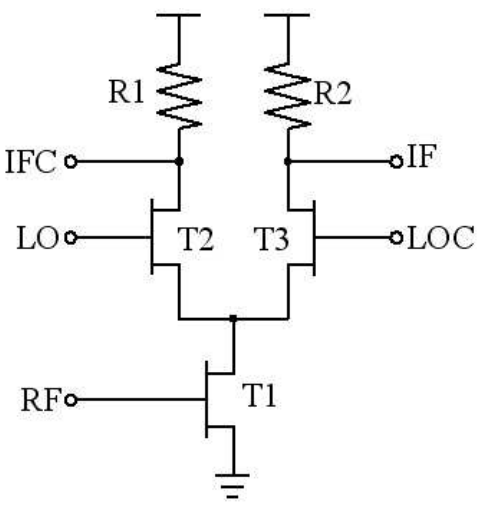

Figure 4 : Schematic of the single-balanced active mixer circuit realized in SiGe HFET technology

The design does not use any frequency selective sections like matching or pole-compensating elements, which results in poor input and output match. However, it allows for the operation of the mixer over a wide range of RF frequencies. Load resistors are chosen to be $200 \Omega$. The presented circuit uses a single metal layer process which excludes the possibility of realizing crossovers as well as MIM capacitors. Due to this technological limitation, several restrictions are imposed on the mixer circuit: 
First, the circuit topology cannot exceed a certain level of complexity. For instance, only a half Gilbert cell can be realized to avoid crossovers. Also, the differential LO signal is generated off-chip due to the lack of capacitors. Second, transistors whose source is not connected to ground can only be realized with a single gate finger in order to eliminate the need for interconnection of transistor source terminals. This imposes a restriction on the total gate width in order to maintain high process yield. Also, it decreases the device's high-frequency performance due to increased gate series resistance. As a compromise with gain and linearity considerations, $100 \mu \mathrm{m}$ gate width transistors have been employed in the present design. Figure 5 shows the layout of the mixer. A redundancy for choosing the drain load resistors is contained. The IC is designed for on-wafer measurement and employs coplanar probe pads with $100 \mu \mathrm{m}$ pitch. Chip dimensions are pad-limited with a total size of $700 \mu \mathrm{m} \times 560 \mu \mathrm{m}$.

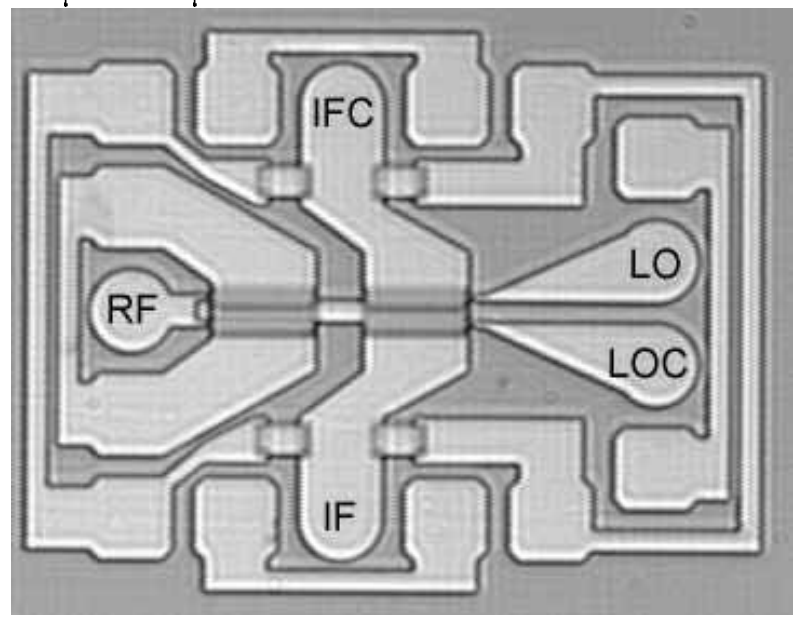

Figure 5: Microphotograph of the SiGe HFET mixer MMIC

For the merit of a simple circuit topology, the differential stage was not de-coupled from the $50 \Omega$ measurement environment via source follower stages. Therefore, the load impedance seen by the differential stage is the DC load resistor in parallel with $50 \Omega$. Simulations indicate that conversion gain could be boosted significantly if source follower stages were included to increase the load impedance.

More complex mixer prototypes making use of a second metallization layer are currently being processed. They will include source follower- as well as on-chip singleended to differential conversion stages.

\section{EXPERIMENTAL RESULTS}

The frequency translating measurement is performed with the Rohde\&Schwarz ZVK vector network analyzer. Cable losses are taken into account and all power levels are corrected to the probe-tip reference plane. The differential LO signals are generated via an external BALUN which adds an additional $1 \mathrm{~dB}$ loss to the applied LO power. Intermediate frequency power is measured single-ended, with the second output port (IFC) being terminated by a $50 \Omega$ impedance. As a consequence, $3 \mathrm{~dB}$ need to be added to the measured conversion gain to describe the differential situation.

Bias conditions are $5 \mathrm{~V}$ supply voltage, a $\mathrm{V}_{\mathrm{GS}}$ of $-0.2 \mathrm{~V}$ for maximum transconductance of the amplifying transistor and $1.2 \mathrm{~V}$ gate voltage for the switching transistors.

At DC, the realized devices show an increased parasitic contact resistance of the drain and source implant areas, while above some corner frequency, the device characteristics take on their initial values. As a result, effective gate-source bias voltages of the transistors are reduced, leading to an onset of conversion gain saturation at lower LO power levels, but also to a reduction in gain. The model accounts for this effect by parallel R-C networks at the drain and source terminals of the device. The values of the increased DC contact resistance and the bypassing capacitance can be extracted from DC-IV and gain versus frequency $\left(\mathrm{S}_{21}\right)$ characteristics.

Conversion gain is found to be $-4.0 \mathrm{~dB}$ and $-4.7 \mathrm{~dB}$ when down-converting $3.0 \mathrm{GHz}$ and $6.0 \mathrm{GHz}$ RF signals, respectively, to an intermediate frequency of $500 \mathrm{MHz}$ using high-side injection of an LO power of $5 \mathrm{dBm}$ (Figure 6). Simulation accurately predicted the conversion gain for all LO power levels, including the linear amplification region, the "knee" region where the LO transistors start acting as switches as well as the saturated switching regime.
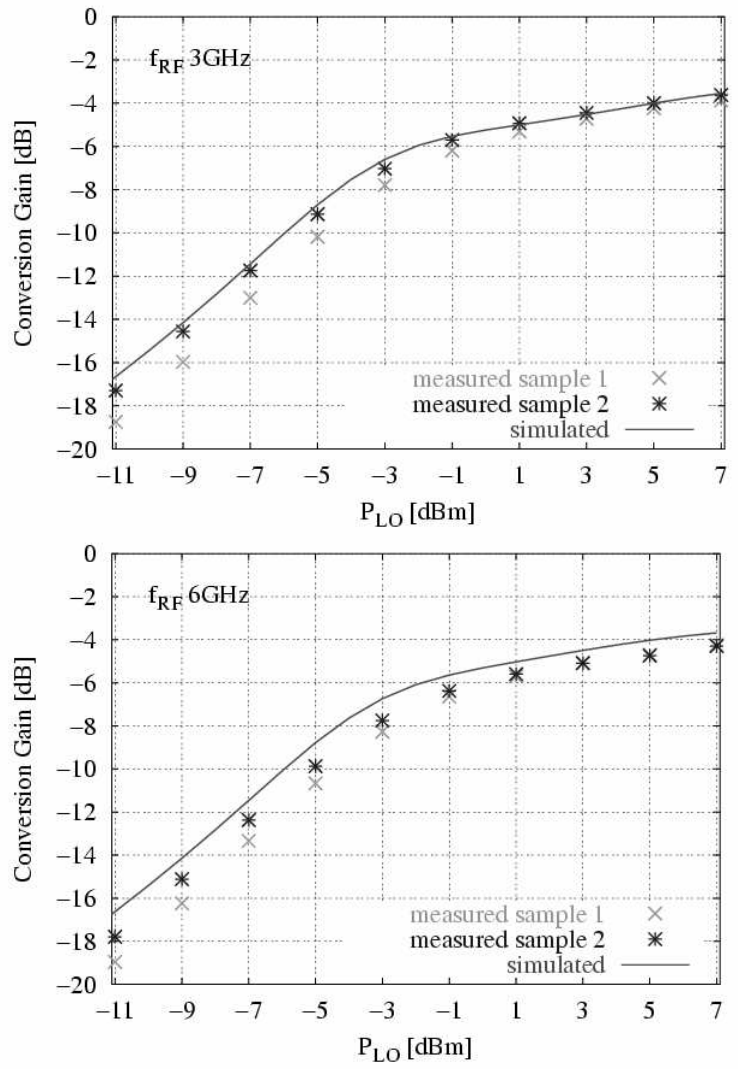

Figure 6: Conversion Gain versus $L O$ power for downconversion of $3.0 \mathrm{GHz}$ (above) and $6.0 \mathrm{GHz}$ (below) signals to $500 \mathrm{MHz}$ IF. RF power is $-20 \mathrm{dBm}$. 
Sweeping the applied RF power at $\mathrm{f}_{\mathrm{RF}}=6 \mathrm{GHz}$ yields an input related $1 \mathrm{~dB}$ compression point of $-8.8 \mathrm{dBm}$ (Figure $7)$. Table 1 summarizes the measured and simulated results.

\begin{tabular}{|c|c|c|}
\hline $\mathrm{f}_{\mathrm{RF}}$ & Measurement & Simulation \\
\hline $3 \mathrm{GHz}$ & $-9.5 \mathrm{dBm}$ & $-10.0 \mathrm{dBm}$ \\
\hline $6 \mathrm{GHz}$ & $-8.8 \mathrm{dBm}$ & $-10.8 \mathrm{dBm}$ \\
\hline
\end{tabular}

Table 1: Measured and simulated 1dB compression points (input related)

The mixer exhibits less than $8 \mathrm{~dB}$ conversion loss for RF frequencies up to $10 \mathrm{GHz}$ (Figure 8).

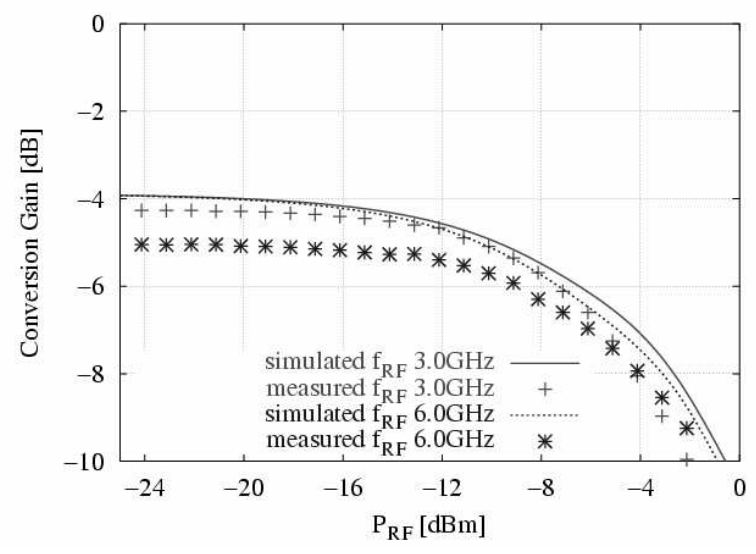

Figure 7: Conversion Gain versus RF power. IF 500MHOOz $L O$ power $5 \mathrm{dBm}$ (HSI).

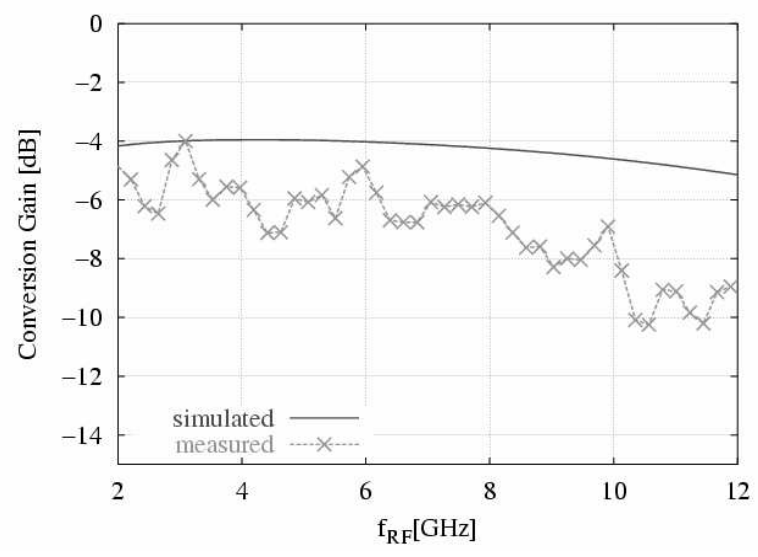

Figure 8: Conversion Gain versus RF frequency for downconversion to $500 \mathrm{MHz}$ IF.LO power is $5 \mathrm{dBm}$ (HSI).

\section{CONCLUSION}

On the basis of a new large-signal simulation model, the first SiGe HEMT active mixer IC has successfully been designed, processed and characterized. In good agreement with simulation predictions, the mixer exhibits a conversion loss of $4.0 \mathrm{~dB}$ for down-conversion of a $3.0 \mathrm{GHz}$ RF signal to an intermediate frequency of $500 \mathrm{MHz}$. At $6 \mathrm{GHz} \mathrm{RF}$, conversion loss is $4.7 \mathrm{~dB}$. These results, obtained from the first manufactured wafers and the high model quality are encouraging for the design of more sophisticated mixer structures like e.g. doublebalanced Gilbert cells including source follower stages. Further improvement is expected from the inclusion of matching networks for operation at specific frequencies.

\section{ACKNOWLEDGEMENT}

Financial support through European Commission's SIGMUND IST-1999-10444, German BMBF Ultra2 13 N7900 as well as UK EPSRC HMOS projects is gratefully acknowledged.

\section{REFERENCES}

[1] M. Zeuner, T. Hackbarth, M. Enciso-Aguilar, F. Aniel, H. von Känel, "Sub 100nm Gate Technologies for Si/SiGe Burried Channel RF Devices", Jap. Appl. Phys., vol. 42, pp. 2363-2366, 2003

[2] M. Enciso-Aguilar, F. Aniel, P. Crozat, R. Adde, H.-J. Herzog, T. Hackbarth, U. König, H. von Känel, "DC and high frequency performance of a $0.1 \mu \mathrm{m}$ n-type $\mathrm{Si} / \mathrm{Si}_{0.6} \mathrm{Ge}_{0.4}$ MODFET with $\mathrm{f}_{\max }=180 \mathrm{GHz}$ at $300 \mathrm{~K}$ and $\mathrm{f}_{\max }=230 \mathrm{GHz}$ at 50K”, El. Letters, vol. 39, pp. 149-150, 2003

[3] M. Zeuner, T. Hackbarth, G. Höck, D. Behammer, U. König, "High-Frequency SiGe n-MODFET for Microwave Applications", IEEE Microwave and Guided Wave Letters, vol. 9, pp. 410-412, October 1999

[4] K. Ismail, J.O. Chu, M. Arafa, "Integrated Enhancementand Depletion-Mode FET's in Modulation-Doped Si/SiGe Heterostructures", IEEE El. Dev. Letters, vol. 18, no. 9, pp. 435-437, Sep. 1997

[5] M. Saxarra et. al., "Transimpedance amplifier based on Si/SiGe MODFETs", Electronics Letters, vol. 34, No. 5, pp. 499-500, March 1998

[6] I. Kallfass, M. Zeuner, U. König, H. Schumacher, T. J. Brazil, ,A Model for SiGe MODFETs with Improved Large-Signal Quality and Frequency Range“, Proc. GaAs Symposium, EuMW September 2002

[7] M. Zeuner, U. König, „SiGe HFET Technology“, Workshop Advanced Silicon Technology, EuMW September 2001

[8] V. I. Cojocaru, T. J. Brazil, “A Scalable General-Purpose Model for Microwave FETs Including DC/AC Dispersion Effects", IEEE MTT, vol. 45, pp. 2248-2255, December 1997

[9] G. Dambrine, A. Cappy, F. Héliodore, E. Playez, "A New Method for Determining the FET Small-Signal Equivalent Circuit", IEEE Trans. MTT, vol 36, no 7, pp. 1151-1159, Jul 1988

[10] M. Berroth, R. Bosch , "High-Frequency Equivalent Circuit of GaAs FET's for Large-Signal Applications", IEEE Trans. MTT, vol. 39, no 2, pp. 224-229, Feb 1991 